\title{
41BX171: A Late Nineteenth Century City Dump in San Antonio, Texas
}

Clive Luke

Follow this and additional works at: https://scholarworks.sfasu.edu/ita

Part of the American Material Culture Commons, Archaeological Anthropology Commons, Environmental Studies Commons, Other American Studies Commons, Other Arts and Humanities Commons, Other History of Art, Architecture, and Archaeology Commons, and the United States History Commons

Tell us how this article helped you.

This Article is brought to you for free and open access by the Center for Regional Heritage Research at SFA ScholarWorks. It has been accepted for inclusion in Index of Texas Archaeology: Open Access Gray Literature from the Lone Star State by an authorized editor of SFA ScholarWorks. For more information, please contact cdsscholarworks@sfasu.edu. 


\section{BX171: A Late Nineteenth Century City Dump in San Antonio, Texas}

\section{Licensing Statement}

This is a work produced for the Texas Department of Transportation (TxDOT) by the report producer. TxDOT and the report producer jointly own all rights, title, and interest in and to all intellectual property developed under TXDOT's contract with the report producer. The report may be cited and brief passages from this publication may be reproduced without permission provided that credit is given to both TxDOT and the report producer. Permission to reprint an entire chapter, section, figures or tables must be obtained in advance from either the Supervisor of the Archeological Studies Branch, Environmental Affairs Division, Texas Department of Transportation, 125 East 11th Street, Austin, Texas, 78701 or from the report producer. 
41 BX 171: A LATE NINETEENTH

CENTURY CITY DUMP IN

SAN ANTONIO, TEXAS

\author{
A PRELIMINARY REPORT \\ by \\ CLIVE LUKE
}

Texas Highway Department Publications in Archeology: 1974 

of these furnaces led to the formation of a special committee which recommended in 1894 that the unused quarry area in the northern part of San Antonio be utilized as the municipal dumping ground. Although no specific beginning date for the dump could be found in the records, 1895 appears to be within reason: a date which is substantiated by archaeological remains. The completion of a large incinerator in 1923 near Salado Creek on the east side of the city may mark the end of dumping in the vicinity of the archaeological site, although dumping and burning may have continued in the area for some years thereafter. Land in the quarry area was sold to the San Antonio Independent School District in 1939 for construction of the Alamo Stadium precluding dumping and burning at the site at that time. A map seen in the City Engineer's Office, San Antonio, indicates that an incinerator was at one time located near the proposed highway right-of-way. The City Engineer's Office does have some information pertaining to City incinerators, but as these documents are not indexed or catalogued, the possibility of locating specific data relative to the dump and incinerator is indeed remote.

One trench was excavated to a length of thirty-five feet. It was oriented approximately north-south and designated Trench One. To eliminate unnecessary man hours, a backhoe initially removed the disturbed and late fill from the upper levels of the trench. Beneath the levels removed by backhoe, the trench was staked in units of five feet by five feet. Each unit was excavated by hand in arbitrary one foot levels, but the ashy soil was not screened. The elevation of a point on a concrete slab in the middle of the trench was established, using a Texas Highway Department Bench Mark as reference. Having so established a point of known elevation, 
corresponding levels of every unit could be kept at a constant elevation.

In addition to testing this dump, a test was made of a second area where core samples demonstrated the presence of garbage. A backhoe trench was used to expose that deposit which was determined to be from the 1940's. No further effort was expended in that area.

A detailed analysis and study of the artifacts at this time seems inadvisable because the sample is so small in terms of the entire dump. Any attempt to utilize the data recovered in September and October 1974 would probably result in biased conclusions. Consequently, further excavation is necessary to provide a larger sample. However, in order to indicate what sort of artifact return was generated, a catalogue of artifacts prepared by Marshall Eiserer, of the Texas Highway Department Archaeology Section, is appended. 


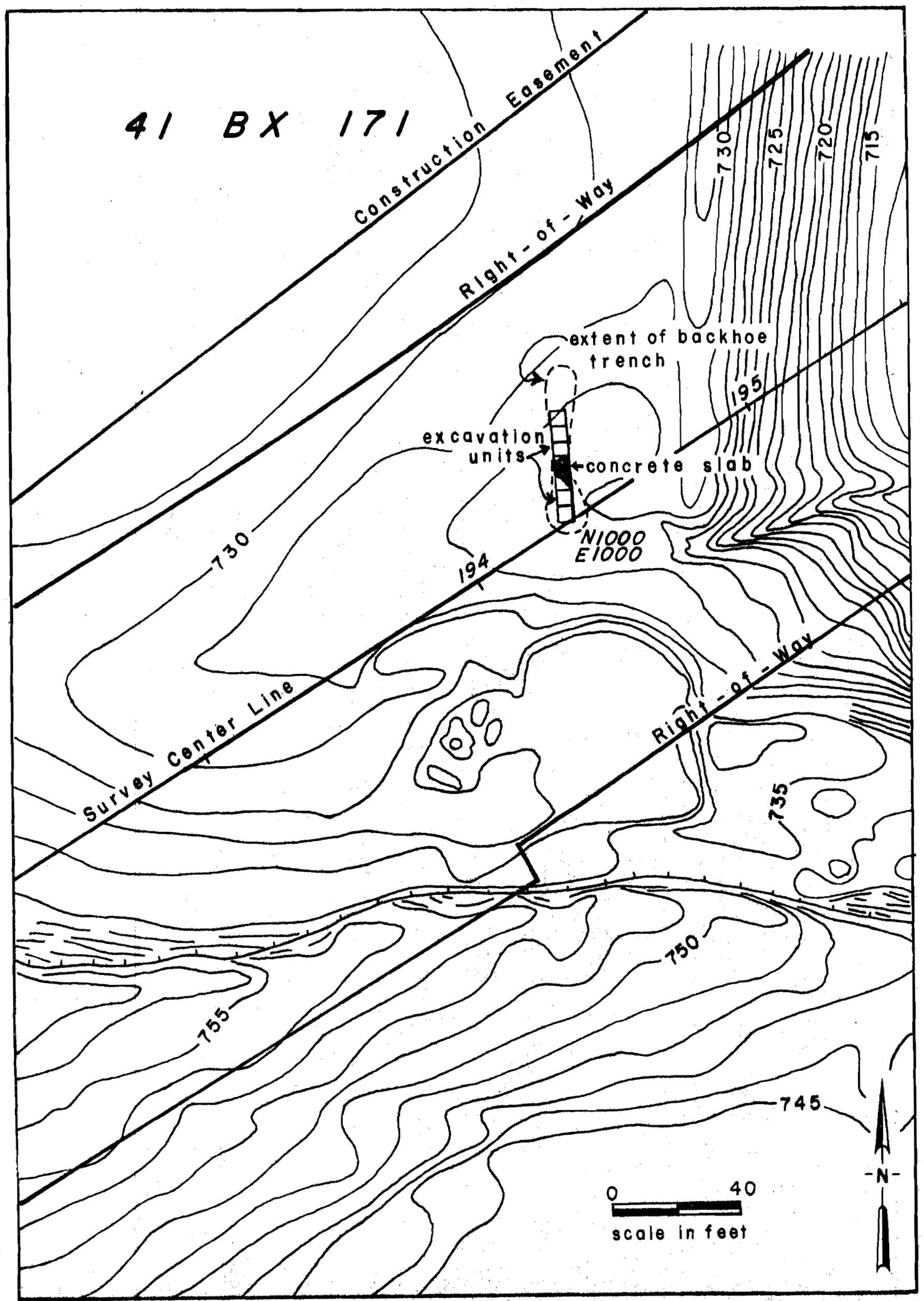

Fig. 1 


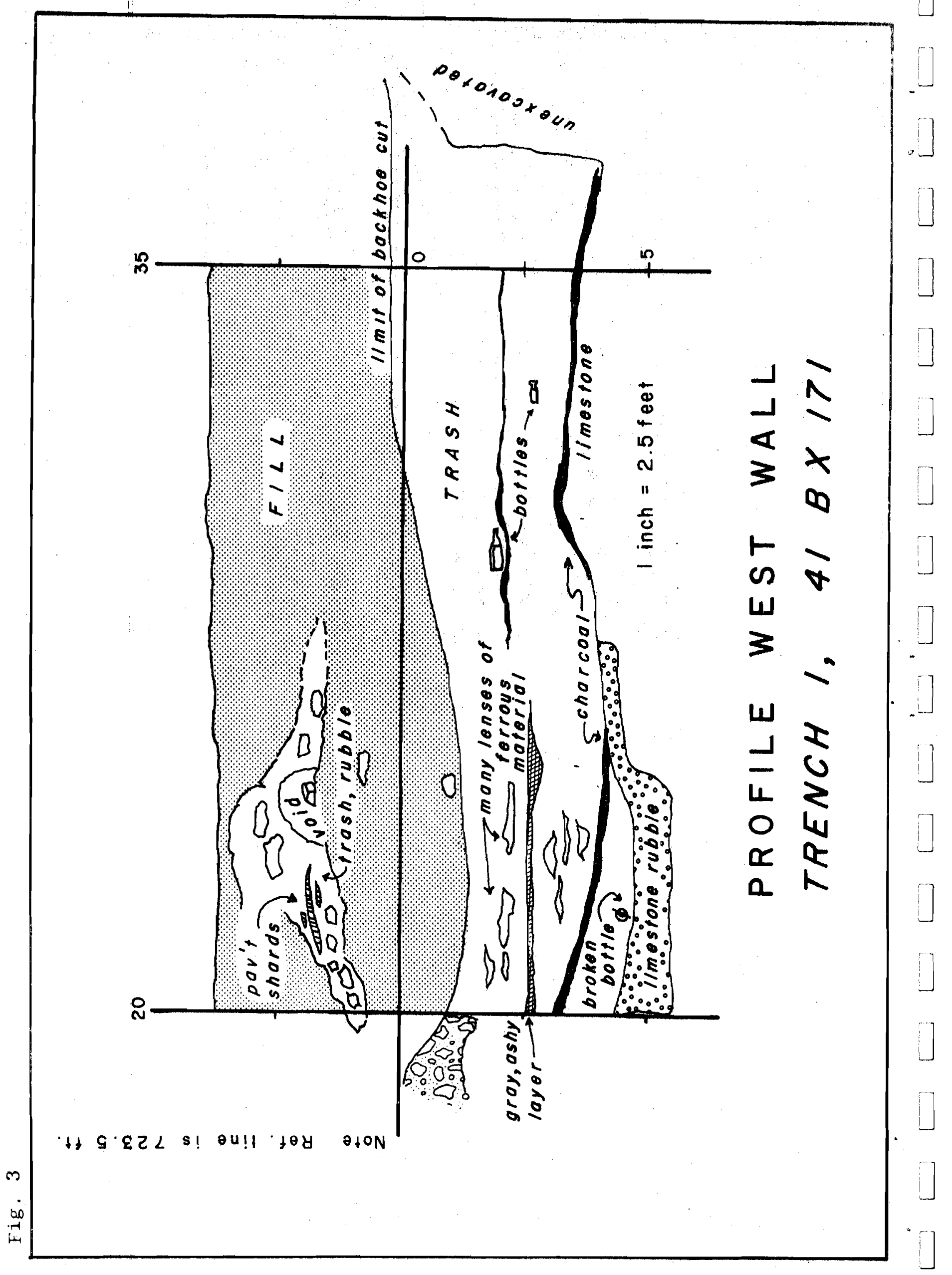




\section{Appendix I \\ Marshall Eiserer}

$41 \mathrm{BX}$ 171: Artifact Inventory by Lots

LOT 1

Provenience: N 995/E1000 to N 1012/E 1000; 0.0' - 6.0'

(Disturbed Fill)

GLASS

BOTTLES

1 Dr. Pitchers Castoria

1 Mrs. Winslows Soothing Syrup

1 Kelly and Palland Pharmacists, El Paso, Texas prescription Bottle

1 Fragment, Wykoffs Union Blueing

1 Neck Fragment of Probable Whiskey

1 Neck Fragment of Probable Hood's Sarsaparilla

1 Neck Fragment Probably Ayers Sarsaparilla

1 Unidentified Light Amber Round Bottle

2 Unidentified Clear Round Bottles

1 Unidentified Cobalt Blue Round Bottle

1 Neck Fragment Probable Wine

1 Cosmetic Jar - Milkglass

GLASSWARE

2 Bases of Clear Goblets

1 Base of Cosmetic Container Opaline Glass

1 Base of Drinking Glass Ruby Glass on Light Amber Glass

MISCELLANEOUS GLASS

1 Insulator Manufactured by Broakfield

CERAMICS

STONEWARE

4 Fragments, 2 Meyer Pieces

CHINA

5 Plate Fragments

1 Marked Pearl White Goodwin Bras.

1 Marked Ironstone China Guaranteed

1 Saucer Fragment

6 Miscellaneous Decorated China Fragments

1 Fragment Probable Handled Sugar Bowl

1 Cup Marked Scholz' (Garden) 


\section{MISCELLANEOUS CERAMICS}

1 Mouth Fragment Gin Bottle

2 Pieces of Glazed Flower Vases or Pots

1 Basal Fragment of Arnold Ink Container Vitreous Stone Bottles, J. Bourne \& Son Denby by Potter

1 Porcelain Top Stopper for Lightning Bottle Stopper

\section{METAL}

IRON

1 Lock

1 Horseshoe

1 Unidentified Object, possible clock 


\section{BX 171}

LOT 2

Provenience : N1000/E1000, Level 1 (Disturbed) (Below Lot 1)

\section{GLASS}

\section{BOTTLES}

1 J. M. Clark Pickle Co. Bottle, Light Amber Shoulder \& Neck Fragment

2 Crown Stopper Bottles - Probable Beer

1 Neck \& Mouth Amber Bottle

1 Neck of Possible Peppersauce Bottle - Clear

1 Shoulder Fragment - Possible Beer

1 Fragment Prickly Ash Bitters Bottle

2 Pieces Dark Green Stained Glass

MISCELLANEOUS GLASS

1 Goblet Fragment

1 Stopper

1 Button

\section{CERAMICS}

STONEWARE

2 Pieces Stoneware

1 Unglazed

CHINA

12 Pieces Miscellaneous China

FAUNAL

4 Oyster Shell 
41 BX $1 / 71$

LOT 3

Provenience: South Dump

6 Fragments of Bottle Glass

2 Aqua Coca Cola Bottle Glass

Design Patented 1915

1 Clear Fragment Whiskey Bottle

Machine Made - post 1903

$[P]$ in t Embossed on Side

2 Miscellaneous Clear Fragments 
41 BX 171

LOT 5

Provenience: N1000/E 1000, Level 1 (Undisturbed)

\section{GLASS}

BOTTLES

1 Fragments of Chamberlain's Cough Remedy

1 Complete Bromo Seltzer, Emerson Drug. Co.

1 Body Fragment "American Pickles (Tennessee Style)"

1 Clear Neck, Prescription Ware Bottle

1 Unidentified Aqua Shoulder \& Neck Fragment (Possible Shoe Polish)

1 Milkglass Porcelain Liner for Mason Style Fruit Jar Embossed Consolidated Fruit Jar Company, New York

\section{CERAMICS}

STONEWARE

1 Shoulder Fragment Stoneware

CHINA

3 Miscellaneous Fragments China

1 Cup Fragment (China)

\section{MISCELLANEOUS CERAMICS}

1 Door Knob (White) 
$41 \mathrm{BX} 171$

LOT 6

Provenience: N1000/E1000, Level 2

\section{GLASS}

\section{BOTTLES}

1 Basal Sections of Green Demijohn 2 Gallon Bottle

1 Shoulder Fragment Mellins Infant Food

1 Mouth Probable Whiskey Bottle

1 Mouth Hutchison Soda

1 Base Probable Wine Bottle

1 Bottle Mrs. Winslows Soothing Syrup

1 Homeopathic Tube Vial

11 Miscellaneous Pieces

1 Base Bottle Curtice Brothers Preserves MISCELLANEOUS GLASS

1 Fragment of a Schlur Palm Garden Salon Goblet

1 Base of Goblet

4 Pieces Dark Green Stained Glass

1 Opaline Glass Fragment

1 Lamp Shade Frag

1 Pressed Glass Bowl Fragment

CERAMICS

STONEWARE

3 Stoneware Fragments

2 Mexican Looking Piece of Pottery

CHINA

1 Child's Toy Saucer or Plate

$10 \mathrm{Miscellaneous} \mathrm{China} \mathrm{Sherds}$

2 Ironstone Sherds (Trade Marks)

4 Decorated Sherds

3 Cup Fragments

2 Bowl Fragments

1 Earl White, Goodwin Brothers Plate Fragment

MISCELLANEOUS CERAMICS

1 Possible Pipe Stand

1 Corner Tile 
LOT 6 (Continued)

MISCELLANEOUS

1 Shoe Heel

1 Toothbrush Handle

1 Unidentified Brass or Copper Object, Probably a Plug

1 Piece Slate

1 Carson Battery, Core, Debossed - Solar 
41 $\mathrm{BX} 171$

LOT 7

Provenience: N1005/E1000, Level 1 (Under Lot 1)

\section{GLASS}

BOTTLES

1 Base San Antonio Coca Cola Bottling Co.

1 Dr. Pitchers Castoria Bottle

1 Shoulder \& Neck Portion Wyckoffs Union Blueing

1 Fragments Jo-Jo Liquor Flask

1 Milkglass Fragment

\section{CERAMICS}

STONEWARE

2 Glazed Sherds

1 Unglazed

CHINA

1 Plate Royal Stone China Baker \& Co.

1 Royal Ironstone, Johnson Eng, (Bowl)

7 Decorated Sherds

22 Miscellaneous Sherds

1 Blue Sherd (Handle)

1 Cup Fragment

IRON

1 Horseshoe

\section{MISCELLANEOUS}

1 Comb Fragment

1 Battery Core 
41 BX 171

LOT 8

Provenience: N 1005/E1000, Level 2

GLASS

BOTTLES

1 Unidentified Clear Bottle

(Sirup Reconstituant a Larseniate De Fer

Soluble de a Clermont)

1 Unembossed Prescription Ware Bottle (Buffalo Oval)

1 Fragmented Scotts Emulsion "Cod Liver Oil"

1 Badly Heated Mason Type Preserves Jar

1 Basal Portion Probable Wine Bottle

4 Unidentified Pieces of Bottles

MISCELLANEOUS GLASS

1 Fragment Window Pane

1 Lamp Chimney Rim

1 Glass Fragment

1 Goblet Base

\section{CERAMICS}

CHINA

1 Oval Plate K.T.\& K. China

1 Plate Ironstone China Alfred - Meakin England

1 Oval Plate (Ironstone China)

4 Ornament Sherds

18 Unidentified Sherds

MISCELLANEOUS CERAMICS

2 Inside Glazed Sherds

2 Fragments of Pipe

MISCELLANEOUS

1 Battery Core

1 Toothbrush Handle

1 Stoneware Fragment

1 Graphite Washer 
41 BX 171

LOT 9

Provenience: $\quad$ Backdirt from Backhoe Trench \#1

\section{GLASS}

BOTTLES

1 Mouth and Neck Fragment Clear Liquor Flask

MISSCELLANEOUS GLASS

1 Glass Stopper, was Cork Wrapper

\section{CERAMIC}

CHINA

1 Base Fragment of Plate: Hanley, England J\&C M eakin Semi-porcelain

MISCELLANEOUS CERAMICS

1 Teakettle Spout

1 Dog Body

1 Rookwood Pottery Piece Base Fragment 
41 BX 171

LOT 10

Provenience: Backhoe Trench \# 1, Section 2

\section{GLASS}

BOTTLES

1 Sloan'in Liniment (Machine Made) Post 1903

1 Wine $1 / 2 \mathrm{Pint}$

1 No Deposit No Return Dr. Pepper Bottle

1 Beer A.B.G.M. Co.

1 Probable Peppersauce (Clear)

2 Duerler San Antonio, Texas (Crown) Soda

1 Probable Steak Sauce

1 Coca Cola San Antonio, Texas Before 1915

1 Lea \& Perkins Wochestershire Sauce

1 Body Fragment Soda or Beer

1 Wittemore Boston (Shoe Polish)

1 Probable Shoe Polish

1 Sanfords Black Ink (Sm Co.)

1 Probable Shoe Polish Machine Made Post 1903

1 E R. Durkee \& Co. (Flavoring)

1 Alamo Drug Store, A Dreiss San Antonio, Texas Prescription Bottle

1 Unidentified Bottle EJB (Bitters) (?)

1 Mucilage (B e 11)

1 Sample (?) Masons International A 1 Sauce

1 Perfume or Cologne (?)

1 Homeopathic $\mathrm{V}$ i a l

1 Probable Shoe Polish

1 Young American Lintment D6 Brown New York

2 Bromo Seltzer

1 Quart Amber Beer R \& Co.

1 Tabasco Sauce (?)

1 Spice Bottle HR\&B Thurben \& Co.

3 Unidentified Handmade Bottles

1 Unidentified Machine Made Bottle

1 Clear B ottle Screwcap Sanfords on Base

1 Jar Armour Packers on Base (Cheese) (?)

GLASSWARE

5 Goblet Bodies

1 Mug

MISCELLANEOUS GLASS

1 Unidentified Milkglass Object Embossed Osborn Mfg. Co. New York

1 Fragment Window Pane 
LOT 10 (Continued)

CERAMICS

STONEWARE

10 Base Fragment; Many Appear to be Meyer

5 Handles, 2 Meyer

1 Spitoon Fragment

1 Denby Pottery Fragment (Ink)

CHINA

1 Bowl Scholz's Garden

1 Server (?) Royal Patent Ironstone

1 Plate Base - Hanley

1 Plate Scholz's Garden

1 Saucer - Leonard Vienna Austria

1 Bowl Base (?) The Wheel Pottery Co.

1 Pitcher Base (?) Royal B o n Chrysanthemum Germany

3 Miscellaneous Decorated Sherds

2 Miscellaneous Sherds

MISCELLANEOUS CERAMICS

1 Mortar

2 Brawn Door Knobs

1 Ceramic Spool Insulator

1 Doll Arm

\section{RO N}

1 Single Tree Ring 
Provenience: N1020/E1000 Level 1 (Under Lot 10)

\section{GLASS}

BOTTLE

1 Unidentified Cobalt Blue Bottle

1 Ed Pinvad Bottle Cologne

1 Prescription Bottle C. Schasse Druggist 277 West Commerce St. San Antonio

1 Unidentified Bottle C.B. Wood(?)\& Sons, Rochester,

1 Mouth Aqua Glass Unidentified

1 Beer Bottle Neck and Mouth, Quart

1 Fragment Mason Jar

MISCELLANEOUS GLASS

1 Piece Blue Glass

1 Golden Bead or Button

1 Goblet Stem \& Base

1 A Scholtz Palm Garden Goblet Fragment

1 Glass Bowl Fragment

CERAMICS

STONEWARE

4 Miscellaneous Stoneware

4 Unidentified Sherds

MISCELLANEOUS CERAMICS

1 Bisque Doll Body, Head Missing

1 Oriental Toothpaste Container 
41 BX 171

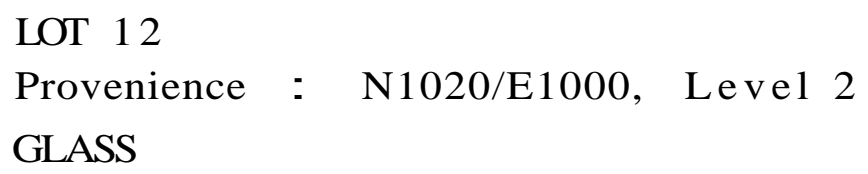

1 Body Fragment Probable Wine Basal Frag. - Dr. S. B.H. \& Co. "B itters"

1 Shoulder \& Neck Fragment Thompsons Phosphate of Wild

1 Base Wine Bottle

Cherry

1 Sanfords Black Ink Bottle

1 Neck of Wine Bottle

1 Side Panel Gebhardts Chili Bottle

1 Mouth Hutchison Soda

1 Mouth Prescription B ottle

1 Mouth Beer B ottle

1 Body Sherd Duffys Malt Whiskey

12 Unidentified Sherds

MISCELLANEOUS GLASS

1 Lamp Chimney Rim

1 Fragment Window Pane

1 Fragment Reinforced Glass (Chicken Wire)

1 Button

1 Milk Glass Container

1 Pitcher Fragment

1 "Candy Container" Fragment

1 Lamp Fragment

\section{CERAMICS}

STONEWARE

15 Glazed Sherds

Unglazed Sherd

Denby Pottery Fragment (Ink)

\section{CHINA}

7 Fragments Large Plate or Plate "Stone China T.P.C. Co. Fragments Decorated Saucer Fragments Saucer "Carlsbad" on Base

Dresden ${ }^{1}$ 
LOT 12 (Continued)

5 Cup Fragments

3 Plate Fragments

12 Miscellaneous Sherds

1 Fragment of Creamer

1 Castor

MISCELLANEOUS CERAMICS

1 Clay Pipe

1 Large Unglazed Piece of Pottery (Crock)

METAL

1 Spike (Railroad)

1 Brass Lamp Holder (Wall Lamp)

1 Pearl Button 
41 BX 171

Prvenience: N1020/E1000, Level 3

\section{GLASS}

\section{BOTTLES}

1 Prickley Ash Polkroot Potassium The Great Purifier

2 Bottle Bases, Possibly Porter or Ale

2 Bottle Bases, Possibly Wine

1 Possible Flavoring

1 Cheeseborough Vaseline Bottle

1 Possible Tabasco Sauce

1 Dr. D. Jaynes Carminative Balsam

1 Ponds Extract

1 Aqua Possible Wine Bottle Base

2 Fragments Possible Flavoring or Extract "Ball Neck

1 Body Sherd Possible Hock Wine

Panel ${ }^{\mathrm{v}}$ Type

1 Body Sherd Possible Wine

1 Prescription Bottle Clavin, Druggist, Commerce St.

1 Side Panel Cod Li[verOil] San Antonio

3 Unidentified Mouth Fragments

2 Unidentified Fragments

1 Palmers Perfume or Cologne

\section{GLASSWARE}

5 Bases or Stems of Goblets

\section{MISCELLANEOUS GLASS}

1 Glas Stopper Hutchison Soda

1 Glas Stopper

1 Milk Glass Liner Ball Mason

1 Chimney Rim

\section{CERAMICS}

\section{STONEWARE}

5 Sherds of Stoneware

1 Crock Lid Fragment

2 Unglazed Sherds

1 Gin Bottle Base

1 Denby Ink (Arnold Ink Co.) 
LOT '13 (Continued)

CHINA

1 Fragment M ustache Cup

$17 \mathrm{M}$ iscellaneous Sherds

1 Pitcher B ase The C.C.T.P. Co. Semigranite

1 Possible Pitcher Base

2 Sherds from Possible Pitcher

$1 \mathrm{H}$ andle

1 Base Sherd - Stone China Wilkinson

1 Green Glazed Small Vessel Base - Possible Creamer 1 Deep Saucer K.T\&K., China

\section{MISCELLANEOUS}

1 Toothbrush Handle - Bone, Dupont \& Evers 


\section{BX 171}

LOT 14

Provenience: N1020/E1000, Level 4

\section{GLASS}

\section{BOTTLES}

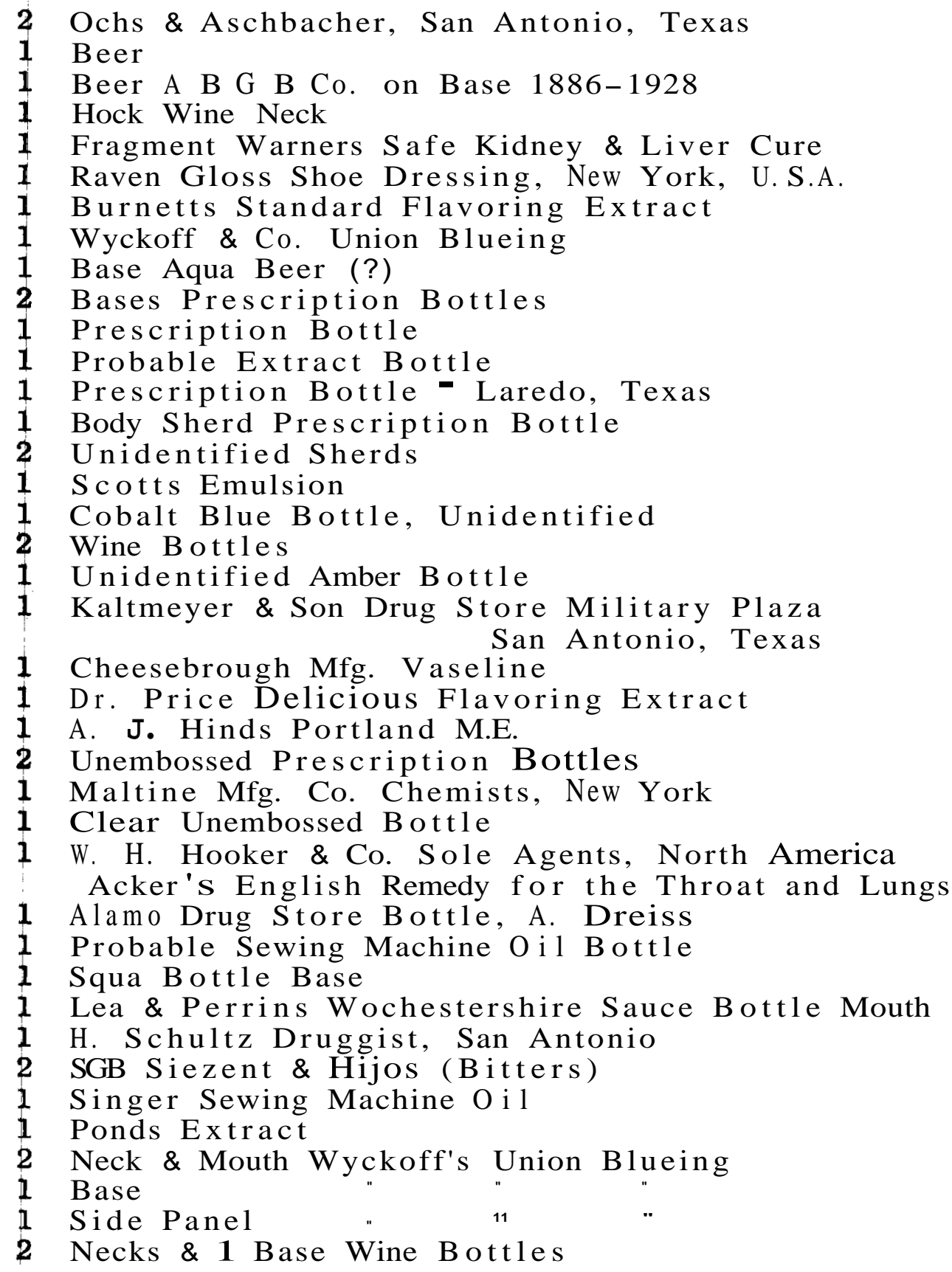


LOT 14 (Continued)

1 Shoulder and Neck of Picnic Flask

1 Shoulder of Hock Wine

1 Hoods Sarsaparilla Bottle Panel

1 Probable Pickle or Relish Bottle (Neck)

1 Bottle Called A Dixie Panel

1 Sample Whiskey

\section{GLASSWARE}

1 Goblet Stem

2 Heavy Glasses

1 Shot Glass

2 Goblets

1 Fragment of Triple Layer Glas Object Probably a

1 Bowl Sherd Glass, Layers Clear on Red on White

1 Chimney Sherd

\section{MISCELLANEOUS GLASS}

1 Probable Compote Stem

2 Sherds Window Pane

\section{POTTERY}

STONEWARE

5 Sherds of Meyer Jug

6 Miscellaneous Sherds

\section{CHINA}

5 Cup Sherds

5 Saucer Sherds

4 Plate Sherds

1 Soap Dish Sherd

1 Urn Sherd

5 Urn Sherds

5 Wash Bowl \$herds

2 Cheese Jar Sherde

1 Flower Pot Sherd

2 Sherds of Cassarole Lids

11 Miscellaneous Sherds

2 Sherds of Pitcher

3 Sherds of Wash Basin

\section{MISCELLANEOUS CERAMICS}

1 Sewer Pipe Sherd 
LOT 14 (Continued)

MISCELLANEOUS

1 Copper or Brass Object

1 Equus $M$ andible Section 
41 BX 171

LOT

Provenience: N1010/E1000, Level 5

\section{GLASS}

BOTTLES

1 Body Large 4 Paneled Bottle "Wisconson Panel"

1 Sherd Picnic Flask

1 Shoulder of "Champagne-Catsup" Screw Cap

1 Neck, Wine

1 Shoulder \& Neck "Shoo-Fly" Flask

1 Body Sherd of Quart Beer

3 Unidentified Sherds

GLASSWARE

2 Goblet Sherds

2 Milkglass Sherds

Candy, or Perhaps Cigar Humidor

1 Pleated Window Glass Sherd

\section{CERAMICS}

STONEWARE

3 Stoneware Sherds, 3 Crock Sherds, 1 Unglazed Sherd

\section{CHINA}

3 Sherds Cassarole 1

3 Bowl Sherds

2 Cup Sherds

4 Saucer Sherds

8 Miscellaneous Sherds Possible Soap Dish Sherd Washbowl Sherd

1 Castor

\section{MISCELLANEOUS CERAMICS}

1 Mexican Pottery Sherd umberous Sherds from Urn

MISCELLANEOUS Teakettle Spout

3 Bones

1 Toothbrush Handle "Mavrey Deschamps Paris "Marjoiaie" 1 Cap for Kerosene Lamp 
41 BX 171

LOT 16

Provenience : N 1025/E 1000, Level 1

. (Under L 100)

GLASS

BOTTLES

1 Carters Ink (Cone)

1 Clear Cone Ink

1 Bromo Seltzer

1 Lea \& Perrins Wochestershire Sauce

1 Wine

1 Unidentified Clear Bottle (Widemouth)

1 Prescription Bottle

1 Durkee Bottle, Patented 1877

CERAMICS

CHINA

1 Toy Plate Sherd

MISCELLANEOUS CERAMICS

1 Do ll Head

1 Castor 
$41 \mathrm{BX} 171$

LOT 17

Provenience: N1025/E1000, Level 2

GLASS

BOTTLES

1 Mrs. Windslows Soothing Syrup

1 Prescription Bottle

1 Duerler, San Antonio, Texas (Soda)

1 Twelve Sided Bottle Base

1 Kickup of Aqua Wine (?)

1 Neck Probably Hood's Sarsaparilla

1 Body Tips and Silver Thorn

2 Wine Necks

1 Picnic Flask Body

1 Sample Mellins Infant Food

1 Souders Elegant Flavoring Extract

1 Probable Ale or Porter Bottle Base

1 Saxlehners Bitterquelle Hunyadi Janos

1 Homeopathic Vial

1 Unidentified Clear Bottle

1 Wine Bottle Shoulder

\section{GLASSWARE}

1 Mug Base

1 Flow Glass Handle

1 Possible Vase Stem

1 Unidentified Footed Clear Glass Object 'Pat. Oct 28..,"

1 Venetian Looking Glass Object

MISCELLANEOUS GLASS

1 Hemingray No. 9 Patent May 2, 1893, (Insulator) (Probably in Disturbed Soil)

\section{CERAMTCS}

STONEWARE

3 Meyer Sherds

8 Miscellaneous Sherds

1 Conduit Stoneware Insulator

CHINA

Sherds from Large Serving Platter

1 Cup Sherd 
LOT 17 (Continued)

1 Saucer Sherd

2 Plate Sherds

2 Sherds from Wash Basin, J.M. \& Son Ironstone -

2 Sherds Bowl Goodwin Pottery

S. Liverpool

2 Sherds From Pitcher

5 Miscellaneous Sherds

1 Little Statue, Head Missing

METAL

1 Horseshoe

1 Muleshoe

1 Soldering Iron (Brass)

1 Unidentified Brass Object

\section{MISCELLANEOUS}

2 Pieces of Slate

1 Piece Coal

3 Pieces of Bone

1 Toothbrush Handle Qualite Supericure Deltamex

Dettre France

1 Piece of Very Recent Braided Cotton Cord 
41 BX 171

LOT 18

Provenience : N1025/E1000, Level 3

\section{GLASS}

BOTTLES

2 Lea \& Perrins Wochertershire Sauce

1 Cuticura System of Curing Constitutional Humors

1 Warners Safe Kidney and Liver Cure

1 M. Golden's Leather Cement

1 Shoulder of "Pot" Mustard Bottle

1 Shoulder of Preserve Ware

14 Paneled Clear Bottle "Wisconsin" Style

1 Bromo Seltzer

1 Prescription Bottle "Baltimore Oval"

1 Eclipse Styler Olive O i 1

1 Small "Garretts" Snuff

1 Beer Aqua B.G. Co. on Bade Burlington Glas s Co., Canada

1 Johann Hoff Liquor

1 Beer, Amber, R\& Co. on Base, Make Unknown

1 Whittlemore Boston U.S.A. (Shoe Polish)

1 Square of American Style Pickle Bottle

1 Preserve Possibly Brandied Peach Bottle (Green)

1 Square Unembossed Aqua Bottle "Square P olish"

1 Capers Pepper Bottle (Neck)

1 Round Aqua Bottle Possible Polish

1 Cobalt Blue Round Bottle (Neck), Unidentified

1 Shoulder of Aqua Quart Beer

2 Wines

1 Singer Sewing Machine O il Bottle (Body)

1 Maltine Mfg. Co, Chemist New York Bottle

1 Unidentified Small Clear Square Bottle

1 Rubifoam for the Teeth (Tooth Powder)

1 Wm. Appmann Druggist San Antonio, Texas

1 Section of Possible Blueing Bottle

1 Portion of Clear Cone Ink

1 Unidentified Bottle Base Fragment

GLASSWARE

2 Glass Bases

5 Goblet Sherds

1 Stem of Possible Compote

1 Sherd of Glass Bowl 
LOT 18 (Continued)

MISCELLANEOUS GLASS

1 Window Pane Sherd

1 Chimney Rim

\section{CERAMICS}

STONEWARE

1 Gallon Jug

5 Miscellaneous Stoneware Sherds

\section{CHINA}

1 Washbowl (Sherds)

7 Plate Sherds

2 Cup Sherds

1 Doll Head

1 Bisque Doll Head

1 Ornate Saucer

1 Toy Cup

1 Toy Saucer

1 Plate Brighton \& Concourse Souvineer Plate

\section{MISCELLANEOUS CERAMICS}

1 Doll leg

METAL

1 Kerosene Lamp Burner

1 Brass Toy (Wagon and House)

2 Unidentified Brass Objects

MISCELLANEOUS

1 Fragment of Slate

2 Pieces Cut Bone

1 Bone

1 Cork 


\section{$41 \quad B X \quad 171$}

LOT 19

Provenience: N1025/E1000, Level 4

\section{GLASS}

\section{BOTTLES}

2 Vaseline Cheesebrough $\mathrm{Mfg} . \mathrm{Co}$.

1 Mrs. Winslow's Soothing Syrup

1 Atrask's Magnetic Balsam

3 Hock Wine Fragments (Same Bottle)

1 Bitterquelle Saxlehner's Hunyadi Janos

1 Base Fragment Cathedral Hexagon Pickle

1 Preserve

1 prescription Bottle, Kalteyer \& Schuchard, E. Houston.

1 Wine Bottle Light Green

San Antonio

1 Wine Bottle Dark Green

1 Cone Ink

1 Insectine Trademark

1 Shoulder and Neck Wine Bottle

1 Neck Quart Beer

1 Sanford Black Ink

3 Preserve or Square Pickle Bottles

1 Possible Sewing Machine Oil Bottle

1 Green Bottle Neck

2 Mason Style Fruit Jar Mouth

1 Small Unembossed Prescription Bottle

1 Dr. Hostetters Stomach Bitters

1 F. Bachrach San Antonio (Soda Water)

1 Ochs \& Aschbacher San Antonio, Texas (Soda Water)

1 Hutchison Soda Mouth

1 Prescription Bottle, Tips \& Silverhorne, Prescription Druggist, San Antonio, Texas

1 Possible Peppersauce Bottle (Tabasco)

1 Shoulder of Picnic Flask (Liquor)

1 P Moller, 011 Ecor Cador ver Christiania (Unidentified)

5 Miscellaneous Sherds

1 Jar, au Roi des Gourmets, Paris

1 Hoods Sarsaparilla

\section{GLASSWARE}

5 Goblets

\section{MISCELLANEOUS GLASS}

1 Pressed Glass Cover

3 Sherds of Window Glass

1 Lightning Stopper Milkglass

1 Button 
LOT 19 (Continued)

\section{CERAMICS}

STONEWARE

4 Meyer Sherds

3 Miscellaneous Sherds

1 Unglazed Sherd

1 Flower Pot Base

1 Mexican Sherd

CHINA

1 J.B. Thorn Chemist London John Tarrant Sole Agent for New York, Some Type of Cream Jar

1 Saucer Scholz's Garden

39 Miscellanedus Sherds

2 Saucer Sherds

8 Plate Sherds

3 Cup Sherds

2 Bowl Sherds

1 Pitcher Sherd

1 Wash Bowl, Many Sherds

MISCELLANEOUS CERAMICS

3 Doll Heads, 1 Bisque

1 Insulator

METAL

1 Horseshoe

1 Group of Copperwire

MISCELLANEOUS

2 Bones

1 Battery Core Carbon Rod

1 Wad of Paper 
41 BX 171

LOT 20

Proveniehce: Removal of Disturbed Fill from Balk

GLASS

BOTTLES

1 Bromo Seltzer, Emerson Drug Co. Baltimore

1 Dr. Prices Delicious Flavoring Extract

1 Davis Vegetable Pain Killer

1 Duerler, San Antonio, Texas; SB\&G Co. on Base

Streator Bottle \& Glass Co. (1881-1905)

GLASSWARE

1 Goblet

CERAMICS

1 Cobalt Blue Glazed Marble

METAL

1 Wagon Wheel Hub 


\section{$41 \mathrm{BX} 171$}

LOT 21

Provenience: N 1030/E1000, Level 1

GLASS

BOTTLES

1 Base of Probable Wyckoff ' sUnion Blueing

1 Neck "American" Style Preserve Bottle

1 Mucilage Bottle

1 Body of Prescription Bottle

1 Side Panel "Chamberlain Med. Co."

1 Possible Mustard Jar

GLASSWARE

2 Goblet Sherds

1 Probable Salt or Pepper Shaker

1 Jigger

MISCELLANEOUS GLASS

1 Sherd Construction Glass (Pleated)

\section{CERAMIC8}

STONEWARE

1 Stoneware Sherd

CHINA

1 Porcelain Plate Sherd

M I SCELLANEOUS

1 Oyster Shell 


\title{
41 BX 171
}

LOT 22

Provenience: N1030/E1000, Level 2

GLASS

BOTTLES

13 Paneled Bottle "Missouri" Paneled Bottle

1 Section Chamberlain's Cough Remedy

1 In k

1 Prescription Bottle Aug. Schweppe $(f x$

Crescent Drug Stone, San Antonio, Texas

A M P \& Co. on Base; Adelbert M. Foster \& Co.

Chicago 1895-1911

\section{MISCELLANEOUS GLASS}

1 Milkglass Button

\section{CERAMICS}

1 Porcelain (White) Doorknob

1 Saucer or Butter Dish, Royal Stone China, Maddoek \& Co.; Burleson, England

1 Small Goose Statue

\section{MISCELLANEOUS}

\author{
1 Battery Core (Carbon) \\ 1 Sherd of Slate \\ 2 Pieces of Rubber Hose \\ 1 Piece of Cotton Rope, Maybe part of a Harness \\ 1 Piece of Wetstone
}




\section{BX 171}

\section{LOT 23}

Provenience: N1030/E1000, Level 3

GLASS

\section{BOTTLES}

1 Acker's English Remedy for the Throat \& Lungs

1 Silverthorn \& Wagner Prescription Druggist, San Antonio

2 The Evans Chemical Company, Proprietors, Cincinnati, Ohio

1 Syrup of Figs, California, Fig. U.S.A. Arup Co., San

1 Unembossed Round Bottle Unidentified

Francisco, California

3 Clear Unidentified Sherds

1 Hutchison Type soda Base ... hrach... Antonio, Texas

1 Base Large Green Wine Bottle

1 Prescription Bottle, Alamo Drug Store, San Antonio

1 Cone Ink

1 Sharp \& Dohme, Caltimore (Medicine)

1 Prescription Bottle; Buffalo Oval

1 Olive Oil

1 Hoyts German Cologne; W. Hoyt \& Co., Lowell, Mass.

1 Dr. King, New Discovery for Consumtion

1 J.M. Clark Pickle St. Louis English Pickle or

Chow-Chow "M issour i" Styler B ottle

14 Paneled Extract Bottle "Tall Ball Neck Panel"

1 Prescription Bottle Golden Gate Oval

1 Large Amber Bottle Neck

1 Unidentified Bottle Embossed ...lappe, Apothekenbesize ..rindorf

Bottles with Labels

1 Porter

1 Sanfords Blacking

1 Bixby Shoe Polish

GLASSWARE
1 Glass
1 Goblet
1 Goblet Body

\section{CERAMICS}

STONEWARE

3 Meyer Sherds, 1 Meyer Base

1 Crock Sherd

1 Miscellaneous Sherd 
LOT 23 (Continued)

1 Crock Rim Stoneware

2 Porcelain Handles

1 Saucer Sherd

1 Probable Washbowl Sherd

1 Unidentified Object Royal Stone China, Johnson Bros.

1 Pipe "Dutch Import"

CHINA

1 Bowl, KT\&K Co. China

2 Lid Sherds

2 Lid Handles

3 Saucer Sherds

1 Serving Bowl Sherd

METAL

1 Unidentified Iron Object

1 Lead Bar

Miscsllaneous

$\begin{array}{ll}1 & \text { Roast Bone } \\ 3 & \text { Bones } \\ 1 & \text { Shoe } \\ 2 & \text { Pieces Slate }\end{array}$ 


\section{BX 171}

LOT 24

Provenience: N1030/E1000,Level 4

GLASS

BOTTLES

1 Garretts Snuff

1 The Mother's Friend, Atlanta, Ga.

2 Bromo Seltzers

1 Possible Castor Oil

1 Philadelphia Oval (prescription Bottle)

1 Auc Schweppe, Cressent Drug Store

1 Olive Oil

1 Lea \& Perrins Wochestershire Sauce

1 Round Stand Ink

1 Cone Mucilgge (?)

2 Cone Inks

1 Demijohn Mouth and Neck

1 Tricopherus for the Skin and Hair

1 Hoods Sarsaparilla

3 Aqua Wine (?)

1 Prescription Bottle "Golden Gate" Style

1 M. Goldens Leather Cement

1 Dr. Hostetters Stomach Bitters

1 Light Green Wine

2 Dark Green Wine Sherds

1 Hutchison soda

1 Beer Amber Quart

1 Square Clear Unidentified Bottle

1 Atwood and Steele Chemical Works

1 Bixby Shoe polish Mouth

1 Hock Wine Mouth

1 Mrs. Winslow's Soothing Syrup

1 Picnic Flask (Liquor)

1 Ink

1 Possible Mineral Water Bottle

1 Aqua Wine Bottle Base Sherds

1 San Pedro Drug Store, San Antonio, Texas Bottle

1 vaseline, Cheeseburough Mfg. Co.

1 Mason Type Preserve Jar Mouth

1 Sauce or Castor Oil Bottle

1 Homeopathic Vial

4 Unidentified Sherds

1 Jar (Milkglass) Vanilla

1 Pickle Bottle

1 Mucilage Bottle

1 Prescription Bottle (Golden Gate) Style

1 Mustard Bottle 
LOT 24 (Continued)

GLASSWARE

1 Pilsner Glass Anhueser Busch

1 Pilsner Glass San Antonio Brewing Assn.

1 Probable Jelly Tumbler

3 Sherds From Goblets

1 Mug Sherd

1 Mug Handle

2 Glass or Goblet Rim Sherds

1 Milk Glas S Container

1 Pressed Glass Bowl

MISCELLANEOUS GLASS

1 Fancy or Ribbed Chimney Rim

$1 \quad$ Plain Chimney Rim

1 Glass Tube

\section{CERAMICS}

\section{STONEWARE}

1 Unglazed Heavy and Thick Rim Sherd

1 Unglazed Sherd

8 Miscellaneous Sherds, Some possibly Meyer

CHINA

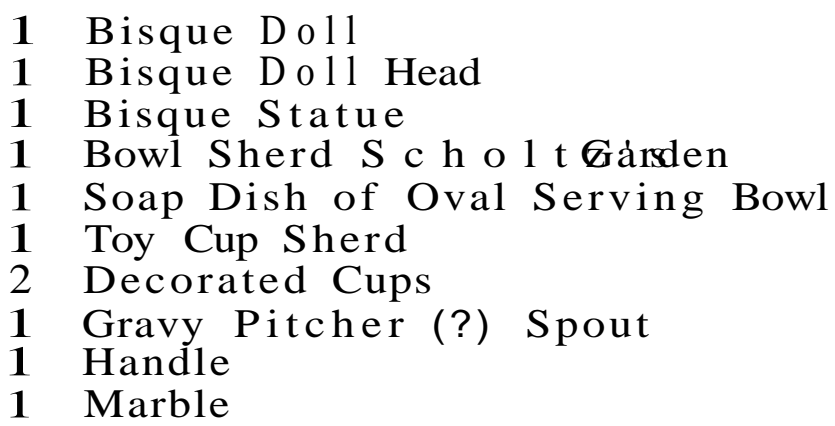

METAL

1 Piece Insulated Copper Wire

1 Piece Steel Wire

1 Piece Iron Pipe

1 Piece Sheet Metal

M ISCELLANEOIJS

1 Unidentified Clay Object

1 Cut Bone

1 Battery Core

1 Sherd Mexican Pottery 
41 BX 171

LOT 25

Provenience: N1030/E1000, Level 5

\section{GLASS}

\section{BOTTLES}

1 Bromo Seltzer

2 Soudens Elegant Flavoring Extracts

1 Burnetts Standard Flavoring Extracts

1 Prescription Bottle (No Embossing)

1 Castor O i 1

1 Possible Mustard Bottle

1 Chamberlain's Cough Remedy

1 Olive O i 1

24 Panel Tall Ball Neck Bottle

1 Hutchison Soda (Sunset Bottling Works) San Antonio, Texas

1 Peppersauce or Castor O i 1

3 Different Sizes Wyckoff's Union Blueing

1 Mrs. Winslows Soothing Syrup

1 Dr. Hawkins Wild Cherry Bitters

2 Beer Bases (Quart)

1 Hutchison Soda

3 Wine Bottle Bases

1 Hock Wine Base

2 Bottle Necks

1 Picnic Flask (Liquor)

1 American Pickle Bottle

1 Lightner Detroit Mich. (Perfume?)

1 Shoulder Fragment; Dr. Hosteters Stomach Bitters

1 Sample Whiskey

10 Miscellaneous Sherds

1 Cold Cream Jar (Blue)

1 Masons Genuine Porcelain Lined Cap (For Mason Jar)

1 Square Unidentified Bottle

\section{GLASSWARE}

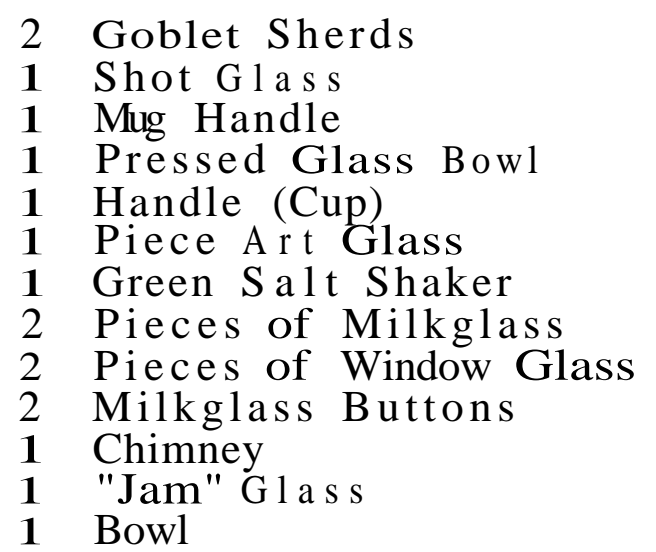


LOT 25 (Continued)

\section{CERAMICS}

STONEWARE

2 Sherds; 1 Jug Base, One Crock Lip

2 Miscellaneous Sherds, 1 - 4 Gallon Meyer Crock

\section{CHINA}

1 Bisque Doll Head

6 Saucer Sherds

8 Plate Sherds

2 Bowl Sherds

2 Cup Sherds

1 Pitcher KT\&K on Base

8 Probable Plate Sherds

2 Lid Sherds

3 Pot Sherds

3 Bowl Sherds

5 Oval Plate Sherds

1 Toy Cup Sherd

50 Miscellaneous Sherds

1 Handled Pot Sherd

MISCELLANEOUS

5 Pieces Sheet Metal

1 Brick Fragment

1 Clothes Pi n

1 Bone

1 Piece of Mexican Pottery 


\section{BX 171}

LOT 26

Provenience: N1030/E1000, Level 6

\section{GLASS}

\section{BOTTLES}

1 Wine Bottle Body

1 Pinuad Bottle (Toilet Water)

1 Quart Picnic Flask (Liquor)

1 Pint Picnic Flask (Liquor)

1 Screw Cap Catsup

1 Pumkin Seed Flask (Liquor)

1 Colgate Perfume Co. Bottle

1 Beer Bottle ABG Co.

1 Coffin Flask (Liquor) Neck

1 Unembossed Prescription Bottle

\section{MISCELLANEOUS GLASS}

1 Chimney Rim

CERAMIC

STONEWARE

1 Meyer Jug

CHINA

1 Doll Head

1 Plate

1 Probable Pitcher

1 Bowl

1 Cosemetic Cap (Maison Dorin, Paris)

1 Possible Doll Cup or Bowl

\section{MISCELLANEOUS}

1 Brass Ring: Possible Pull Knob for Furniture 3 Bones 
$41 \mathrm{BX} 171$

LOT 27

Provenience: N1025/E1000, Level 5

GLASS

\section{BOTTLES}

2 A. Trasks Magnetic Ointment

1 Tips \& Silverthorn Prescription Druggist, San Antonio, Texas

1 Souders Elegant Flavoring Extracts Co., Dayton, Ohio

1 Atwood \& Steele Chicago, Illinois

I Dr. Pitchers Castoria

1 Wickoffs Union Blueing

1 Bitter Quele Hunyadl Janos

1 Quart Amber Beer Neck

1 Lea \& Perrins Wochestershire Sauce

\section{MISCELLANEOUS GLASS}
1 Light Green Glass Lamp Shade
1 Glass Base
1 Handle

\section{CERAMICS}

\section{CHINA}

\section{Saucer Sherde}

2 Plate Sherds

2 Bowl Sherds

1 Castor Sherd

Fragments of Large Washbowl or Urn

\section{MISCELLANEOUS}

\section{Bones \\ 1 Marble}

